\title{
Single fibers of skeletal muscle as a novel graft for cell transplantation to the heart
}

\author{
Ken Suzuki, MD, PhD ${ }^{\mathrm{a}^{*}}$ \\ Bari Murtuza, MA, FRCS ${ }^{a^{*}}$ \\ Louise Heslop, $\mathrm{PhD}{ }^{\mathrm{b}}$ \\ Jennifer E. Morgan, $\mathrm{PhD}^{\mathrm{b}}$
}

Ryszard T. Smolenski, MD, PhD

Noriko Suzuki, MDa

Terence A. Partridge, $\mathrm{PhD}^{\mathrm{b}}$

Magdi H. Yacoub, FRS ${ }^{a}$
From the Department of Cardiothoracic Surgery, Heart Science Centre, Harefield Hospital, ${ }^{a}$ and Muscle Cell Biology Group, Hammersmith Hospital, Imperial College Faculty of Medicine, ${ }^{b}$ London, United Kingdom.

This study was supported by the British Heart Foundation and the Harefield Research Foundation. Ken Suzuki is supported by the research fellowship from the Uehara Memorial Foundation.

Received for publication April 30, 2001; revisions requested July 9, 2001; revisions received Oct 24, 2001; accepted for publication Nov 7, 2001.

Address for reprints: Professor Sir Magdi H. Yacoub, Department of Cardiothoracic Surgery, Harefield Hospital, Hill End Rd, Harefield, Middlesex, UB9 6JH, United Kingdom (E-mail: k.suzuki@ic.ac.uk).

*These authors contributed equally to this work.

J Thorac Cardiovasc Surg 2002;123:984-92

Copyright () 2002 by The American Association for Thoracic Surgery

0022-5223/2002 \$35.00+0 $\quad \mathbf{1 2 / 1 / 1 2 1 5 0 7}$

doi:10.1067/mtc.2002.121507
Objective: Skeletal myoblast transplantation is a promising alternative to treat heart failure. A single fiber, the minimal functional unit of skeletal muscle, retains skeletal myoblasts beneath the basal lamina. When surrounding muscle is injured, myoblasts migrate from the fiber into the damaged area to regenerate muscle. We hypothesized that such isolated fibers could be used as an efficient vehicle to deliver myoblasts into damaged myocardium, resulting in improved cardiac function.

Methods: Living single fibers of rat skeletal muscle were isolated, and their behavior was characterized in vitro. Single fibers were injected into the myocardium (at 4 sites, each receiving a single fiber) of rats in 2 models of heart failure induced either by means of doxorubicin administration or left coronary artery occlusion.

Results: Skeletal myoblasts dissociated from an isolated single fiber, proliferated, and differentiated into multinucleated myotubes in vitro. Within 3 days after grafting in vivo, original fibers provided putative myoblasts and disappeared. At 4 weeks, discrete loci consisting of several multinucleated myotubes were observed. Furthermore, singlefiber transplantation significantly improved cardiac function compared with the control treatment in either doxorubicin-treated hearts (maximum dP/dt, $4013.9 \pm 96.1$ vs $3603.1 \pm 102.3 \mathrm{~mm} \mathrm{Hg} / \mathrm{s}$; minimum dP/dt, $-2313.7 \pm 75.1 \mathrm{vs}-2057.1 \pm 52.4 \mathrm{~mm}$ $\mathrm{Hg} / \mathrm{s}$ ) or ischemic hearts (maximum dP/dt, $3905.6 \pm 103.0$ vs $3572.6 \pm 109.7 \mathrm{~mm}$ $\mathrm{Hg} / \mathrm{s} ;$ minimum dP/dt, $-2336.1 \pm 69.7 \mathrm{vs}-2106.4 \pm 74.2 \mathrm{~mm} \mathrm{Hg} / \mathrm{s})$.

Conclusion: Single-fiber transplantation acts as a vehicle for delivering putative skeletal myoblasts that appear to differentiate into myotubes within the myocardium. This was associated with improved function of failing hearts, suggesting its efficacy as a novel graft for cellular cardiomyoplasty.

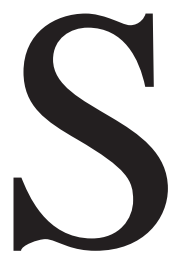

keletal myoblast transplantation is a promising alternative strategy to treat end-stage heart failure. ${ }^{1-4}$ It has been demonstrated that skeletal myoblasts retain the ability to regenerate differentiated functional muscle and to improve cardiac function when transplanted in rabbit and rat heart failure models..$^{3,4}$ In addition, myoblasts can be isolated from patients with heart failure themselves and used as an autograft, obviating the need for immunosuppressive reagents. ${ }^{5}$ Currently, direct intramyocardial injection of a skeletal myoblast suspension is the most popular method to deliver myoblasts into the heart. However, we consider that there remain some important issues that should be addressed to refine this therapeutic strategy for clinical application. One of these is the time-consuming and complex process required to prepare sufficient numbers of purified myoblasts from skeletal muscle. Millions of myoblasts are likely to be required to improve cardiac function by means of direct intramyocardial injection of a skeletal myoblast suspension, even in small 
animals. ${ }^{3,4}$ There are concerns that repeated passaging of primary myoblasts in vitro might cause possible harmful effects, resulting in the alteration of important properties, such as the ability to fuse and differentiate. ${ }^{6}$

Recently, we have established a simple, quick technique to isolate viable single fibers of rodent skeletal muscle. ${ }^{6} \mathrm{~A}$ single fiber (here defined as muscle tissue separated into individual fibers), the minimal functional unit of skeletal muscle, retains myogenic precursors, skeletal myoblasts (satellite cells), between the basal lamina and plasma membrane. The myoblasts are normally mitotically quiescent, but when surrounding muscle is injured, they migrate from the fiber into the damaged area, proliferating and differentiating to regenerate myofibers. ${ }^{7,8}$ In this study we investigated whether transplanted single fibers could work as a vehicle to deliver skeletal myoblasts-myotubes into the myocardium and whether this strategy leads to the improvement of cardiac function in heart failure induced by either doxorubicin administration or myocardial infarction.

\section{Methods}

\section{Animal Care}

All studies were performed with the approval of the institutional ethics committee for animal research and the Home Office. The investigation conforms to the "Principles of Laboratory Animal Care" formulated by the National Society for Medical Research and the "Guide for the Care and Use of Laboratory Animals" published by the US National Institutes of Health (publication No. $85-23,1996)$.

\section{Isolation of Single Muscle Fibers}

Living single fibers of skeletal muscle were isolated according to the previously described method.6,7 Briefly, the extensor digitorum longus of a male Lewis rat (80 g) was removed and digested in $0.2 \%$ type I collagenase (Sigma) in Dulbecco's modified Eagle's medium (DMEM). The muscle was transferred to a petri dish containing serum-free DMEM, in which single muscle fibers were liberated by means of gentle trituration. The individual, intact, viable muscle fibers (approximately $500 \mu \mathrm{m}$ long and $20 \mu \mathrm{m}$ in diameter) were collected and subsequently injected into the heart, as described below.

\section{Characterization of Single Muscle Fibers and Derived Myoblasts}

When cultured in proliferation medium (DMEM with $20 \%$ fetal calf serum, $10 \%$ horse serum, and $0.5 \%$ chicken embryo extract), putative skeletal myoblasts were derived from the fibers and proliferated. Cells were grown to subconfluence in proliferation medium, which was then switched to differentiation medium (DMEM with $2 \%$ horse serum) to induce differentiation. ${ }^{6}$ Cells were incubated to subconfluence to assess the purity of myoblast culture. After fixation and blocking, the samples were incubated in anti$\alpha$-sarcomeric actin monoclonal antibody (Dako) and then in fluorescein isothiocyanate-conjugated secondary antibody (Dako). Nuclei were counterstained with 4,6-diamino-2-phenylindole.
Generation of Doxorubicin- or Myocardial Infarctioninduced Heart Failure

Heart failure was induced by means of doxorubicin administration $^{9,10}$ or left coronary artery (LCA) occlusion ${ }^{11}$ in male Lewis rats $(200 \mathrm{~g})$. Fifty rats were administered with doxorubicin hydrochloride (Sigma) in 6 equal injections (each containing $2.5 \mathrm{mg} / \mathrm{kg}$ in saline solution administered intraperitoneally) over a period of 2 weeks for a total dose of $15 \mathrm{mg} / \mathrm{kg}$. Another 45 rats were anesthetized with sodium pentobarbital $(50 \mathrm{mg} / \mathrm{kg}$ administered intraperitoneally) and mechanically ventilated. Through a left thoracotomy, the LCA was occluded $3 \mathrm{~mm}$ distal to its origin. The surgical wounds were repaired, and the rats were returned to their cages to recover. Aseptic surgical techniques were used throughout the procedure. Rats were observed for 4 weeks after the last injection of doxorubicin or LCA occlusion for their general appearance, behavior, and mortality.

\section{Transplantation of Syngeneic Single Fibers into the Failing Rat Heart}

Four weeks after the last doxorubicin injection or LCA occlusion, appropriately randomized rats were anesthetized and mechanically ventilated. Through a left thoracotomy, single fibers of a syngeneic donor (although not autografts) were injected into the left ventricular (LV) free wall (at 4 sites, each of which was injected with only one fiber in $3 \mu \mathrm{L}$ of DMEM) with a glass micropipette (Drummond Scientific), the end of which was pulled to an extremely sharp point after heating ( $\mathrm{n}=20$ for the doxorubicin model). For the LCA-occluded hearts $(n=18)$, fibers were injected into the border zone of infarcts (not into infarct scar). We believe that injection into the border zone has some potential advantages over the injection into the infarct scar, including easier injection and better blood supply for grafted cells. For the control group, the same volume of DMEM was injected ( $n=20$ for each model). After repairing surgical wounds, the rats were incubated for a further 4 weeks.

\section{Histologic Study}

At 4 weeks after transplantation, hearts from each group were collected, and the left ventricle was cut into 4 segments and frozen in an embedding medium. After fixation, $6-\mu \mathrm{m}$ cryosections were incubated with $0.6 \% \mathrm{H}_{2} \mathrm{O}_{2}$ in $0.1 \%$ Triton $\mathrm{X}-100$. After blocking, the sections were incubated with an anti-skeletal myosin heavy chain (MHC) antibody (Zymed laboratories), which reacts with skeletal MHC but not with cardiac or smooth muscle myosin. This was then incubated with a biotinylated secondary antibody (Dako). The sections were colored with a Dako streptABComplex/HRP kit, according to the manufacturer's instructions, and counterstained with hematoxylin and eosin. Sections were examined by blinded experienced histologists. The number of capillary vessels in the peri-infarct area was counted with a light microscope at 400 magnification by blinded experienced observers on the sections that included injection sites. ${ }^{12}$ Five such high-power fields (HPFs) in each section were randomly selected, and the number of capillaries in each field was averaged and expressed as the number of capillary vessels per HPF $\left(0.2 \mathrm{~mm}^{2}\right)$.

\section{Assessment of Cardiac Function and Infarct Size}

At 4 weeks after single-fiber transplantation, rats $(n=8$ in each group) were anesthetized again as above. After systemic hepa- 
rinization, the hearts were quickly excised and perfused at $1 \mathrm{mH}_{2} \mathrm{O}$ pressure with modified Krebs-Henseleit buffer by using a Langendorff apparatus, as described previously. ${ }^{13}$ After 20 minutes of stabilization, heart rate, ventricular function, balloon volume, and coronary flow were measured with left ventricular end-diastolic pressure (LVEDP) stabilized at $10 \mathrm{~mm} \mathrm{Hg}$ by using a thin-walled balloon. After perfusion, the hearts undergoing LCA occlusion were fixed in $3.7 \%$ neutral-buffered formaldehyde, and the left ventricle was cut into 5 segments parallel to the apex-base axis. A $10-\mu \mathrm{m}$ section from each segment was stained with Masson trichrome and projected onto a screen for computer-assisted planimetry. The ratio of scar length to LV circumferences of the endocardium and epicardium was determined by blinded experienced histologists and expressed as a percentage to define infarct size. ${ }^{11}$

\section{Study for Dynamics of Original Single Fibers}

$\beta$-Galactosidase ( $\beta$-gal)-expressing single fibers of EDL muscle, obtained from a $3 \mathrm{~F}-$-nlacZ-2E transgenic mouse, ${ }^{14}$ were implanted into the hearts of male athymic (nude) rats (150-200 g, Charles River UK Ltd) ${ }^{15}$ to further investigate the dynamics of grafted original fibers after implantation into the heart. The 3F-nlacZ-2E mouse, kindly provided by Dr M. Buckingham (Department of Molecular Biology, Institute Pasteur, France), carried regulatory elements from the locus of the fast myosin light-chain $2 \mathrm{~F} / 3 \mathrm{~F}$ gene that drives a nlac $Z$ reporter gene, and this transgene is expressed by all myonuclei in fast myofibers. ${ }^{14}$ Such $\beta$-gal-expressing single fibers and migrated myoblasts could be more clearly identified in the host myocardium by means of in situ staining for $\beta$-gal. Nude rats were used as recipients to remove the need for immunosuppression. Isolation of single fibers and transplantation into the heart were performed exactly as described above. Heart samples were collected at 1 hour, 1 day, and 3 days after grafting ( $n=3$ at each time point); cut into 4 segments; and frozen in an embedding medium. The samples were cut into $10-\mu \mathrm{m}$ cryosections, fixed in $2 \%$ formaldehyde and $0.2 \%$ glutaraldehyde, and then stained with $1 \mathrm{mg} / \mathrm{mL}$ 5-bromo-4-chloro-3-indoyl- $\beta$-D-galactopyranoside, 5 $\mathrm{mmol} / \mathrm{L}$ potassium ferricyanide, $5 \mathrm{mmol} / \mathrm{L}$ potassium ferrocyanide, and $2 \mathrm{mmol} / \mathrm{L} \mathrm{MgCl}_{2}$ for 12 to 16 hours at $37^{\circ} \mathrm{C} .{ }^{13}$ The sections were counterstained with hematoxylin.

\section{Statistical Analysis}

All values are expressed as means \pm SEM. Statistical comparison of the data was performed by using a Student $t$ test with the Statview version 4.0 statistical package (Abacus Concepts Inc).

\section{Results}

\section{Characteristics of Skeletal Muscle Fibers and Derived Myoblasts}

Putative skeletal myoblasts had dissociated from isolated, living single-fiber explants of Lewis rats within 24 hours after plating (Figure 1, $A$ and $B$ ). Immunocytochemistry demonstrated that all the cells were stained positively for $\alpha$-sarcomeric actin (Figure 1, $C$ ), confirming the high purity of the myoblast culture. The cells were cultured in proliferation medium until subconfluence and then switched to differentiation medium to examine their myogenic differen- tiation ability. Consequently, the cells fused and differentiated into multinucleated myotubes within a week (Figure 1, $D)$.

\section{Histologic Findings after Single-fiber Transplantation}

Discrete loci, positively stained for skeletal MHC, were observed in every doxorubicin-treated heart at 4 weeks after single-fiber transplantation at 27 of the total of 40 injection sites studied under low-power magnification (Figure 2, B). Because this antibody reacts with skeletal MHC but not with cardiac or smooth muscle myosin, these positively stained cells were presumed to be of skeletal myoblast origin. Each of these loci consisted of several discrete, branching, multinucleated myotubes that had aligned with the cardiac fiber axis within native myocardium at the injection site where only one myotube (single fiber) had been implanted (Figure 2, $C$ and $D$ ). Such loci, consisting of several multinucleated myotubes positively stained for skeletal MHC, were also observed locally in the peri-infarct area of every LCA-occluded heart at 4 weeks after single-fiber transplantation at 20 of the 32 injection sites (Figure 3,B). These differentiated, branching, multinucleated myotubes had aligned with the cardiac fiber axis within native myocardium, which is similar to that observed in the doxorubicin-treated hearts (Figure 3, C). Furthermore, angiogenesis induction with mature capillaries was observed in the surrounding area of the grafted myotubes in the single fibertransplanted hearts (Figure 3,D). Local capillary density was significantly $(P=.0046)$ higher in the single fibertransplanted hearts compared with that in the control-treated hearts $(4.1 \pm 0.5$ vs $2.3 \pm 0.2$ vessels/HPF, $\mathrm{n}=8$ for the single fiber-transplanted hearts and $\mathrm{n}=6$ for the controltreated hearts).

\section{Dynamics of Original Single Fibers Grafted Into Myocardium}

$\beta$-Gal-expressing single fibers were implanted into the hearts to further clarify the dynamics of grafted original fibers. In situ staining for $\beta$-gal demonstrated that $\beta$-galpositive surviving single fibers existed in the myocardium 1 hour after implantation (Figure 4, $A$ ) but not in day 1 (Figure $4, B$ ) or 3 (Figure $4, C$ ) samples. At day 1 after grafting, a mass of $\beta$-gal-positive debris, presumably originated from a destroyed single fiber, was observed (Figure 4, $B$ ). At day 3 , such $\beta$-gal-positive debris also disappeared, and instead, $\beta$-gal-positive cells (putative skeletal myoblasts migrated from grafted fiber) were found (Figure 4,C).

\section{Cardiac Function After Single-fiber Transplantation}

The effect of single-fiber transplantation on cardiac function was investigated by using 2 different heart failure models, caused by either doxorubicin administration or LCA ligation. Before the main part of the study, we confirmed 

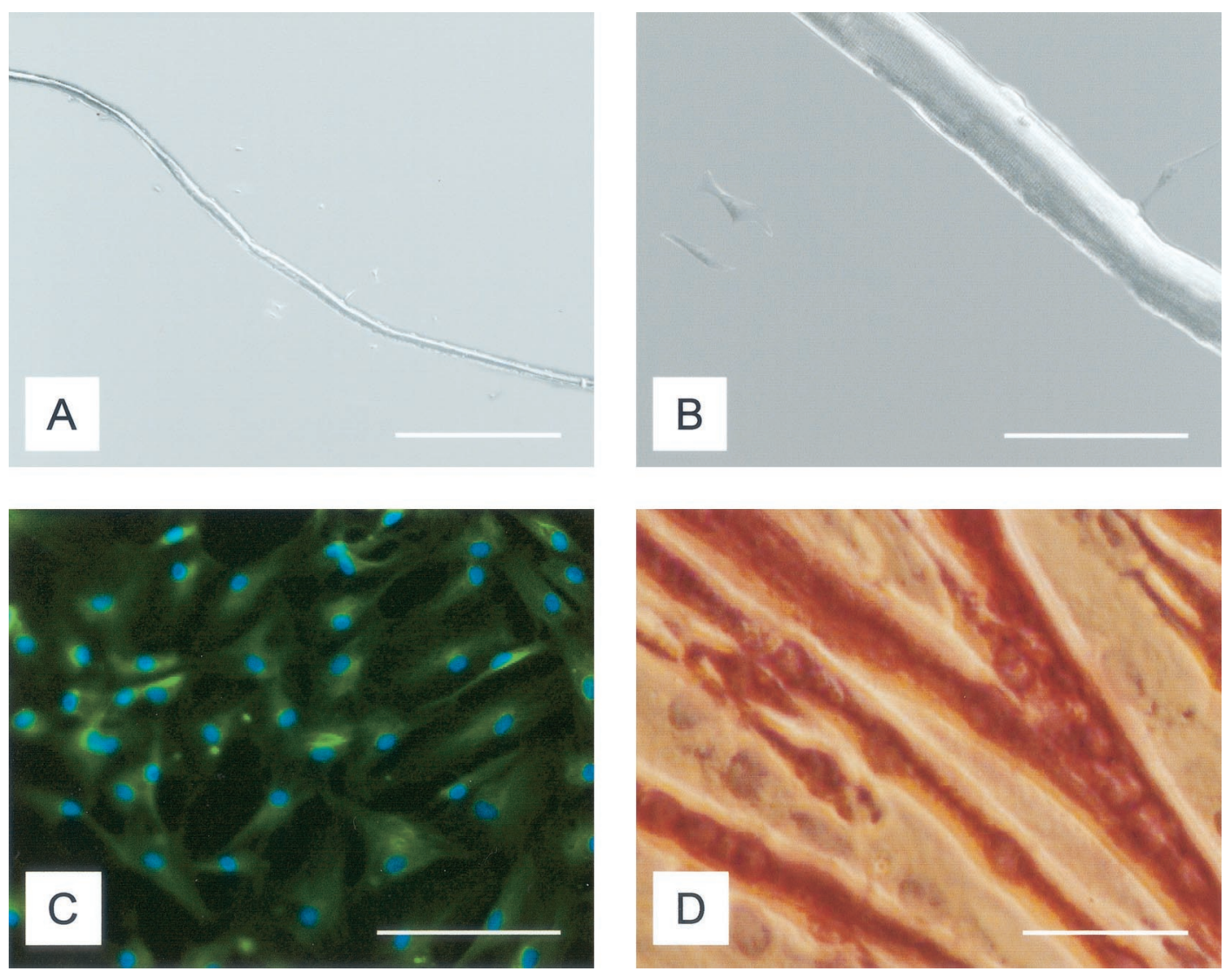

Figure 1. Single muscle fibers and derived myoblasts. A and B, Single fibers were isolated from rat skeletal muscle, from which skeletal myoblasts dissociated within 24 hours after plating (reverse-phase contrast microscopic observation). C, All the derived cells were stained green (fluorescein isothiocyanate) for $\alpha$-sarcomeric actin. Nuclei were counterstained blue with 4,6-diamino-2-phenylindole. D, The dissociated cells fused and differentiated into multinucleated myotubes after switching into differentiation medium. The scale bar indicates $250 \mu \mathrm{m}$ in A and $50 \mu \mathrm{m}$ in B, C, and D.

consistency of functional reduction in these models by using Langendorff isolated heart perfusion. In comparison with the baseline data of normal intact hearts (left ventricular developed pressure [LVDP], $151.6 \pm 2.3 \mathrm{~mm} \mathrm{Hg}$; maximum $\mathrm{dP} / \mathrm{dt}, 4554.3 \pm 50.1 \mathrm{~mm} \mathrm{Hg} / \mathrm{s} ;$ minimum $\mathrm{dP} / \mathrm{dt}$, $-3298.4 \pm 35.4 \mathrm{~mm} \mathrm{Hg} / \mathrm{s}$ at $10 \mathrm{~mm} \mathrm{Hg}$ of LVEDP, $\mathrm{n}=8$ ), cardiac function was significantly reduced at 1 month after doxorubicin administration (LVDP, $124.8 \pm 3.4 \mathrm{~mm} \mathrm{Hg}$; maximum $\mathrm{dP} / \mathrm{dt}, 3631.2 \pm 78.8 \mathrm{~mm} \mathrm{Hg} / \mathrm{s} ;$ minimum $\mathrm{dP} / \mathrm{dt}$, $-2239.6 \pm 46.0 \mathrm{~mm} \mathrm{Hg} / \mathrm{s} ; \mathrm{n}=5, P<.001)$ and LCA ligation (LVDP, $126.9 \pm 3.6 \mathrm{~mm} \mathrm{Hg}$; maximum dP/dt, $3687.4 \pm 84.1$ $\mathrm{mm} \mathrm{Hg} / \mathrm{s} ;$ minimum dP/dt, $-2263.7 .2 \pm 56.2 \mathrm{~mm} \mathrm{Hg} / \mathrm{s} ; \mathrm{n}=$ $7, P<.001)$. Then Langendorff perfusion was performed to assess the functional benefits of single-fiber transplantation 4 weeks after the treatment (Table 1). As regards doxorubicininduced heart failure, LVDP and maximum and minimum $\mathrm{dP} / \mathrm{dt}$ at $10 \mathrm{~mm} \mathrm{Hg}$ of LVEDP were significantly improved in the single fiber-transplanted hearts compared with that seen in the medium-injected hearts. LV chamber size at $10 \mathrm{~mm} \mathrm{Hg}$ of
LVEDP was significantly smaller in the single fiber-transplanted hearts. Similarly, in LCA occlusion-induced heart failure, all of these parameters for both systolic and diastolic function were improved in the single fiber-transplanted hearts associated with higher coronary flow. In addition, these data concerning improved function by means of single-fiber transplantation in LCA-occluded hearts were associated with decreased infarct size in comparison with the that in the control hearts $(36.5 \% \pm 1.5 \%$ vs $43.3 \% \pm 1.8 \%, \mathrm{n}=8, P=.0109)$. Heart rate was not different between the single fiber-transplanted and medium-injected hearts in either heart failure model.

\section{Mortality}

Mortality during the 4-week period after the final doxorubicin injection or LCA occlusion until single-fiber transplantation was $28.0 \%$ (14/50) and $24.4 \%$ (11/45), respectively. Mortality at 4 weeks after single-fiber transplantation was $10.0 \%(2 / 20)$ in the doxorubicin-induced heart failure 

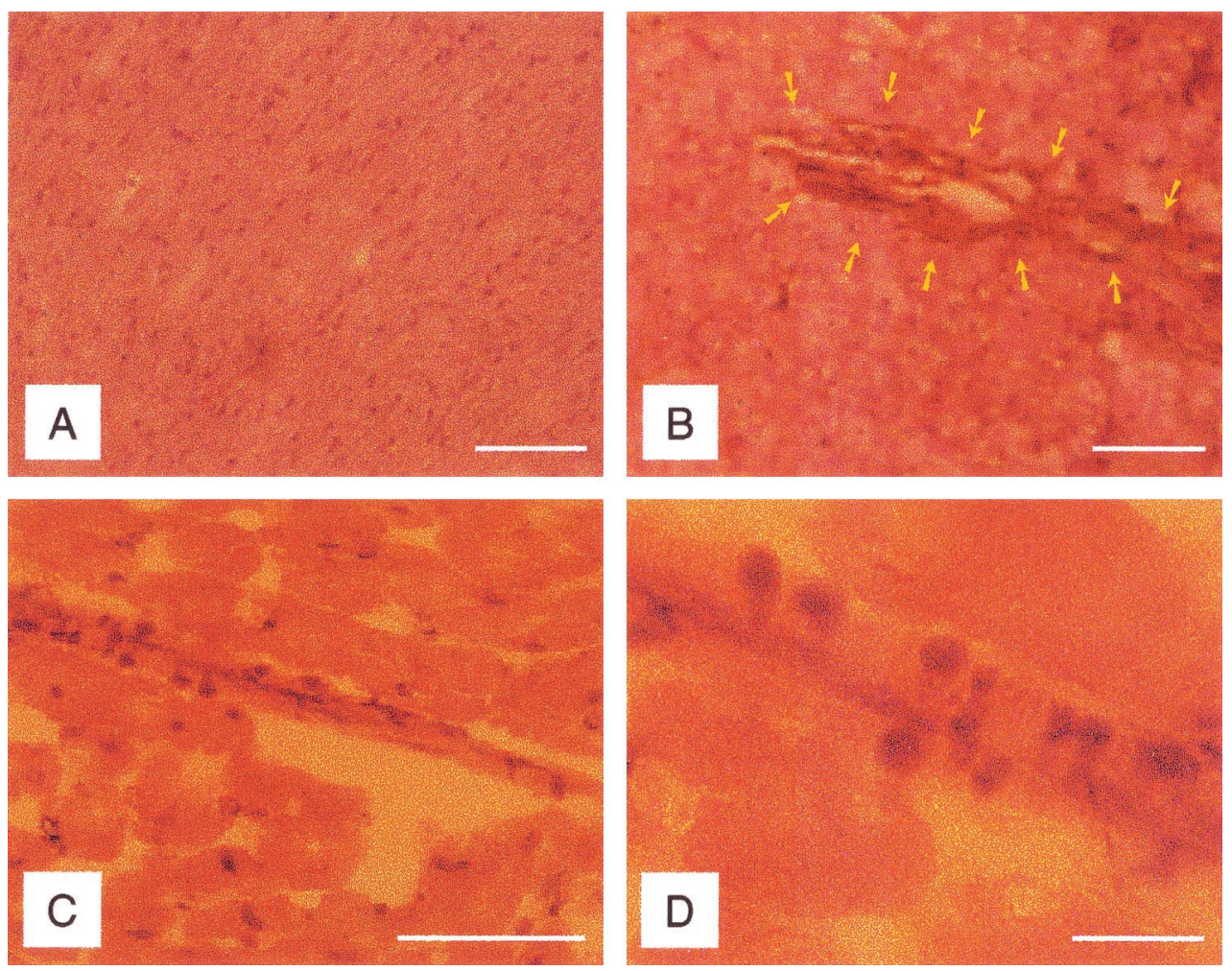

Figure 2. Histologic findings in doxorubicin-induced heart failure. As a result of immunostaining for skeletal MHC (counterstained with hematoxylin and eosin), groups of positively stained, differentiated, multinucleated myotubes (yellow arrows) were observed in the doxorubicin-treated hearts at 4 weeks after single-fiber transplantation (B-D), whereas no cells were stained in medium-injected hearts (A). The scale bar indicates $100 \mu \mathrm{m}$ in A, B, and $C$ and $25 \mu \mathrm{m}$ in $\mathrm{D}$.

model and $11.1 \%(2 / 18)$ in the LCA occlusion model compared with $12.5 \%(2 / 16)$ in the doxorubicin-induced heart failure model and $12.5 \%(2 / 16)$ in the LCA occlusion model after medium injection.

\section{Discussion}

We have demonstrated that single fibers of skeletal muscle grafted to the heart provide putative skeletal myoblasts that appear to differentiate into multinucleate myotubes within the myocardium. This in vivo behavior of grafted single fibers is equivalent to the in vitro observation that has been shown in the present and in other articles. ${ }^{6,7}$ Our study using implantation of $\beta$-gal-expressing single fibers demonstrated that all the grafted original fibers are destroyed and subsequently removed after providing myoblasts into myocardium within 3 days after grafting. We have also described that, 4 weeks after syngeneic single-fiber transplantation, several discrete multinucleated myotubes are formed at each injection site where only one myotube (single fiber) had been implanted. These multinucleated myotubes were branching and appeared to be aligned with the cardiac fiber axis, showing a different appearance from the original sin- gle fiber. Because the mature myonuclei of the original fiber are postmitotic, these data indicates that skeletal myoblasts, which are derived from the parent fiber, are the source of the newly formed multinucleated myotubes.

Furthermore, we have shown that such single-fiber transplantation results in improvement of cardiac function in 2 different types of heart failure induced by either doxorubicin treatment or LCA occlusion, suggesting the feasibility of this strategy for treating heart failure. Both heart failure models used here have been established as stable and reliable models clinically relevant to idiopathic and ischemic cardiomyopathy, respectively. ${ }^{9-11}$ Such consistent significant reduction in cardiac function at 1 month after doxorubicin administration and LCA ligation in comparison with our baseline data of normal intact hearts was confirmed in our own study. A limitation of using the Langendorff perfusion system with isolated hearts is the inability to measure the time course of functional changes in a single animal throughout the pretreatment and posttreatment period. Thus this system would not be optimal to study the degree of functional improvement after single-fiber transplantation 

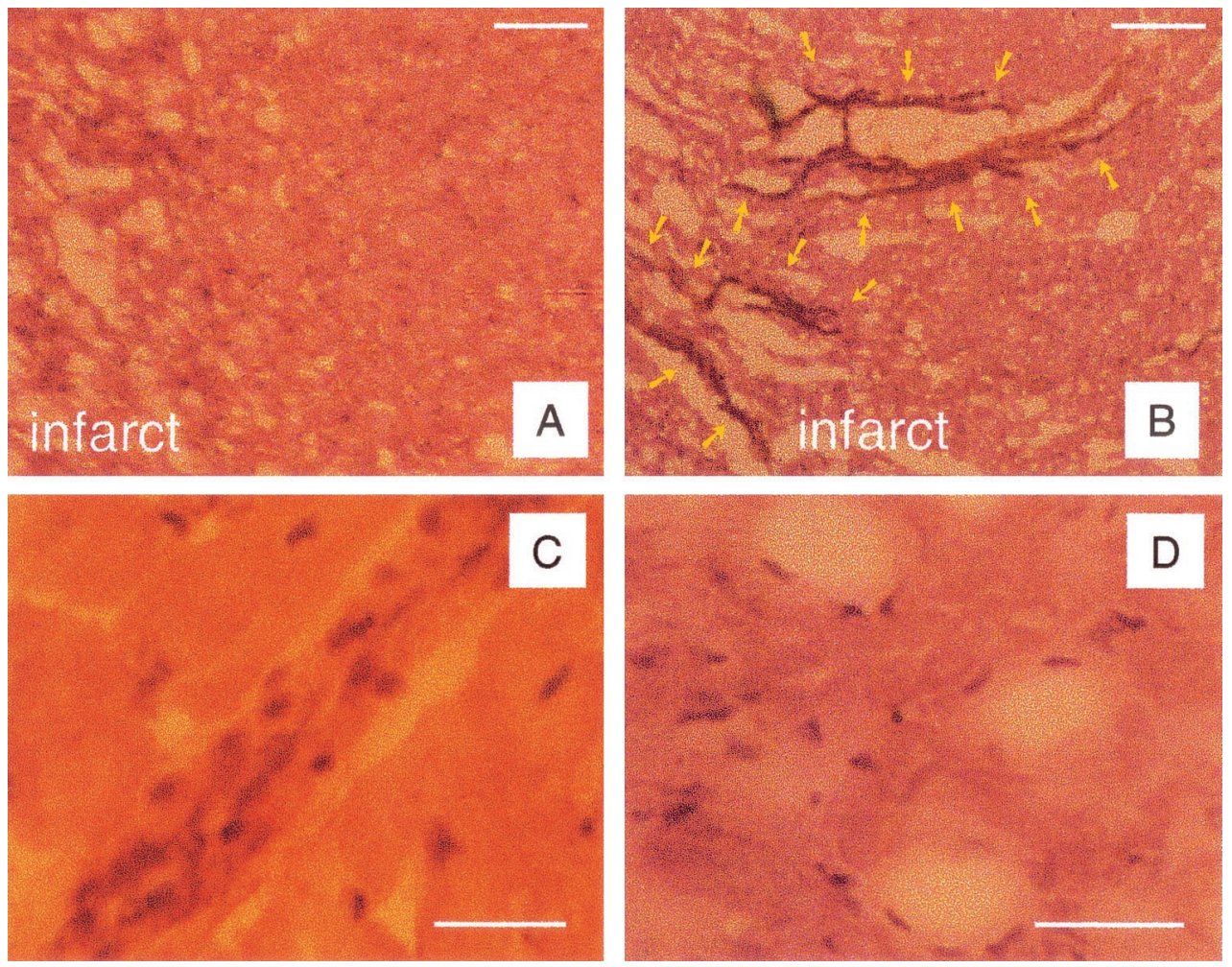

Figure 3. Histologic findings in infarction-induced heart failure. Immunohistochemistry for skeletal MHC (counterstained with hematoxylin and eosin) showed an infarction without skeletal MHC positively stained cells in the LCA-occluded hearts treated by means of medium injection (A). Discrete loci composed of differentiated multinucleated myotubes, which were stained brown for skeletal MHC (yellow arrows), were observed in the peri-infarct area at 4 weeks after single-fiber transplantation (B and C). Angiogenesis with mature capillaries was observed in the surrounding area (D). The scale bar indicates $100 \mu \mathrm{m}$ in $A$ and $B$ and $25 \mu \mathrm{m}$ in $C$ and $D$.

compared with that before transplantation. In this study we therefore focused on the comparison of function between single fiber-treated hearts and sham-treated hearts at the same time point using randomized, diseased rats that had consistent myocardial damage caused by either doxorubicin administration or LCA ligation. As a result, we found a decrease in diastolic compliance in the control-treated hearts compared with that in the single fiber-treated rats in both heart failure models. This might have affected the data of systolic function to some extent; however, it is unlikely that such remarkable improvement both in terms of LVDP and maximum $\mathrm{dP} / \mathrm{dt}$, as demonstrated in our data, resulted from such a difference in diastolic function. Further study is needed to evaluate more detailed functional analysis after single-fiber engraftment, including ventricular chamber size and time course.

The use of single fibers could be advantageous over the conventional method of direct intramyocardial injection of a myoblast suspension in several respects. Foremost, the single-fiber protocol avoids the need for the prolonged in vitro processes required to derive an adequate number of myo- blasts for transplantation. During this several-week period, repeated passaging is likely to be detrimental, and extended incubation carries risks of infection of the primary culture by means of bacteria, fungi, mycoplasma, and viruses. Furthermore, this prolonged in vitro culture might thus cause possible subtle changes in the myoblasts that render them either more visible to the host immune system or less competent for fusion and differentiation in vivo. In contrast, preparations of single fibers can be completed within only a few hours, avoiding the hazards of culture. This feature could be of great significance, particularly in clinical settings. Such easily harvested single fibers would also be promising as grafts in relatively urgent situations. In human patients the use of extensor digitorum longus might not be suitable because single-fiber preparation requires muscle from tendon to tendon. However, there are many other potential sites for harvesting smaller skeletal muscle fibers in human patients, including the digits, which would be clinically applicable. Another advantage of using single fibers is the lesser amount of medium required for injection. A single fiber can be suspended in only 2 to $3 \mu \mathrm{L}$ of 

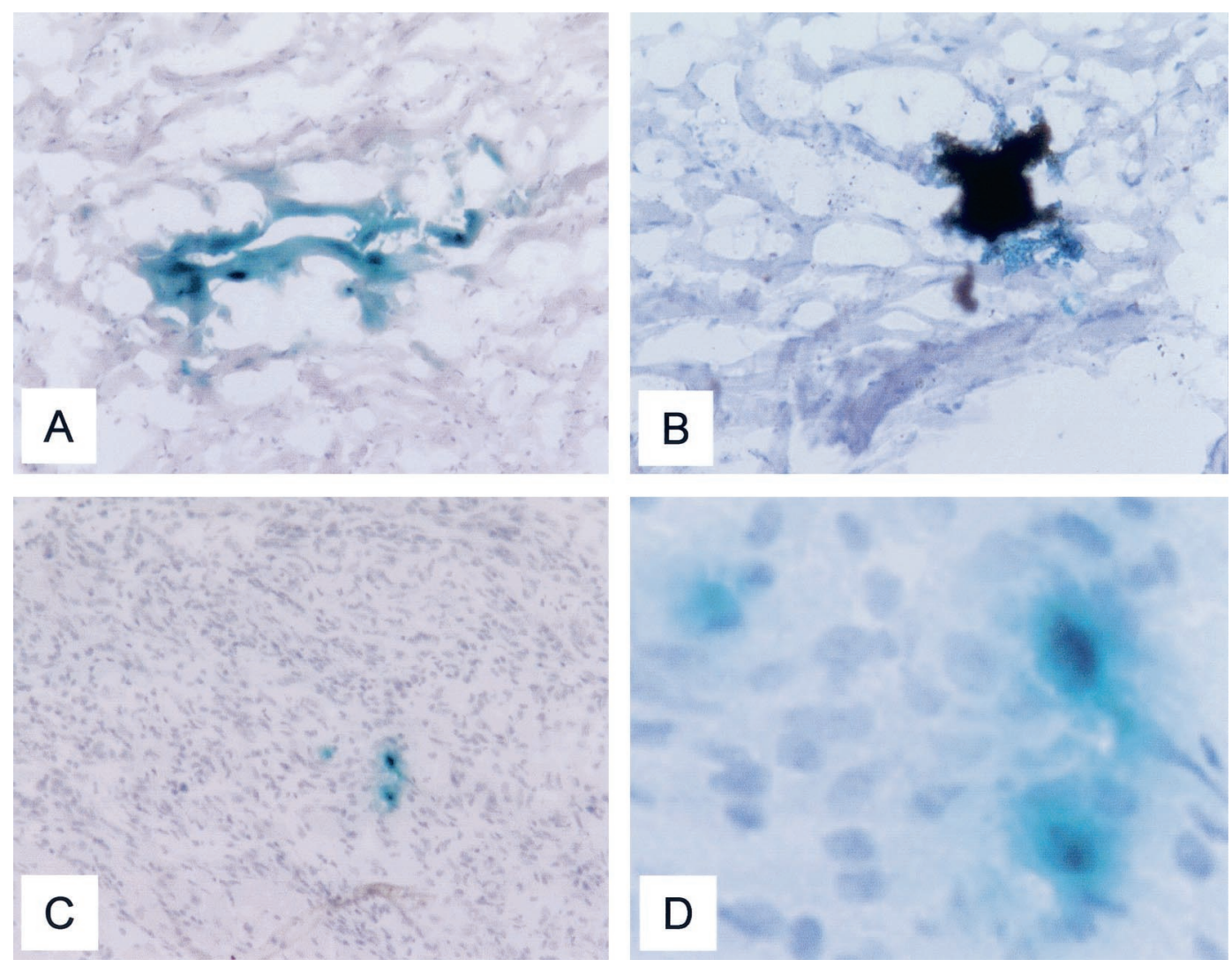

Figure 4. Dynamics of grafted single fibers. $\beta$-Gal-expressing single fibers obtained from a transgenic mouse were implanted into the hearts to clarify the dynamics of grafted original fibers. As a result of in situ staining for $\beta$-gal, $\beta$-gal-positive surviving single fibers were present in the myocardium at 1 hour after grafting (A) but broken into debris at day 1 (B) and completely disappeared at day 3 (C). Instead, $\beta$-gal-positive cells (putative skeletal myoblasts migrated from grafted fiber) were observed at day 3 (C and D).

TABLE 1. Cardiac function in doxorubicin- or LCA occlusion-induced heart failure at day 28 after single-fiber transplantation

\begin{tabular}{|c|c|c|c|c|c|c|}
\hline & HR (beats/min) & LVDP (mm Hg) & $\begin{array}{l}\text { Maximum dP/dt } \\
(\mathrm{mm} \mathrm{Hg} / \mathrm{s})\end{array}$ & $\begin{array}{l}\text { Minimum dP/dt } \\
(\mathrm{mm} \mathrm{Hg} / \mathrm{s})\end{array}$ & $\begin{array}{c}\text { Balloon size } \\
\qquad(\mu \mathrm{L})\end{array}$ & CF $(\mathrm{mL} / \mathrm{min})$ \\
\hline \multicolumn{7}{|l|}{ Doxorubicin } \\
\hline Control & $249.2 \pm 9.9$ & $123.6 \pm 4.0$ & $3603.1 \pm 102.3$ & $-2057.1 \pm 52.4$ & $200.8 \pm 6.7$ & $10.9 \pm 0.5$ \\
\hline Single fiber & $251.4 \pm 9.3$ & $140.6 \pm 5.4$ & $4013.9 \pm 96.1$ & $-2313.7 \pm 75.1$ & $223.1 \pm 6.3$ & $11.0 \pm 0.4$ \\
\hline$P$ value & .9285 & .0240 & .0117 & .0131 & .0286 & .9010 \\
\hline \multicolumn{7}{|l|}{ LCA occlusion } \\
\hline Control & $265.2 \pm 10.8$ & $122.3 \pm 4.3$ & $3572.6 \pm 109.7$ & $-2106.4 \pm 74.2$ & $174.6 \pm 6.3$ & $10.1 \pm 0.4$ \\
\hline Single fiber & $261.9 \pm 11.2$ & $137.5 \pm 5.0$ & $3905.6 \pm 103.0$ & $-2336.1 \pm 69.7$ & $201.4 \pm 8.6$ & $11.8 \pm 0.4$ \\
\hline$P$ value & .9044 & .0363 & .0365 & .0376 & .0257 & .0084 \\
\hline
\end{tabular}

Data were measured at $10 \mathrm{~mm} \mathrm{Hg}$ of LVEDP. Values are expressed as means \pm SEM. $\mathrm{n}=8$ in each group.

$H R$, Heart rate; $C F$, coronary flow; control, hearts infused with serum-free medium; single fiber, hearts grafted with single fibers.

medium. One might expect a lesser injection volume of medium to induce less host myocardial damage as a result of inflammation after cell transplantation. A possible risk of cell transplantation is an embolism caused by grafts leaked into the systemic or coronary circulation. If grafted single fibers leaked, it could easily cause embolism because of their larger size. However, injection of single fibers is not more difficult than injection of cultured cells and requires a 
smaller injection volume, resulting in a possibly lower risk of leakage into the LV cavity or coronary vessels compared with that seen in cell suspension grafting.

In the present study we have shown that as few as 4 single fibers grafted into the heart can improve global LV function. Each single fiber from the Lewis rat extensor digitorum longus retains approximately 20 skeletal myoblasts (satellite cells) according to the results of immunostaining for M-cadherin, a marker for skeletal myoblasts (data not shown), suggesting that the myoblasts derived from these fibers not only survive at a high rate but also retain an adequate ability to proliferate and differentiate within myocardium. A similarly high degree of survival and differentiation is found when isolated mouse single fibers are transplanted into skeletal muscle (data from Heslop and associates, in preparation). Although further investigations are needed to prove this concept in detail, we speculate that using single muscle fibers as a vehicle for delivery of myoblasts might afford natural protective mechanisms to the myoblasts from environmental stress and subsequent adverse pathologic processes, particularly in the early phase after transplantation, which appears to be the most hazardous period for grafted myoblasts. ${ }^{16,17}$ Original single fibers grafted into the myocardium are so sensitive to such environmental stress that they cannot survive for a long time after grafting. Such injury could, at the same time, signal the release of myoblasts, which we believe are the source of the newly formed myotubes observed in our in vivo study.

The report by Scorsin and colleagues ${ }^{3}$ demonstrated that transplantation of 5 million skeletal myoblasts improved LV ejection fraction by $28.9 \%$ (measured by means of echocardiography) in rat ischemic hearts compared with that in medium-injected rats. In the present study transplantation of 4 single fibers improved maximum $\mathrm{dP} / \mathrm{dt}$ by $11.4 \%$ (measured with Langendorff perfusion) in a similar model. This functional improvement seems to be less than half of the previous report; however, these data cannot be compared directly because several critical experimental conditions, including the timing of grafting, are different. In addition, one could expect that transplantation of more single fibers would lead to greater improvement in cardiac function. The extent of skeletal muscle engraftment and the number of skeletal myotubes has not been measured in this study. Although these measurements could be important in explaining enhanced myocardial function, we believe that the quality of the engrafted cells, including proliferation and differentiation capacity, contractile capacity, and gap junction formation ability, could be just as important in mediating improvement. The capacity to secrete growth hormones or cytokines is likely to contribute to improved angiogenesis, as found in our study, or delayed myocardial adverse remodeling, and thus this also might affect the functional result. The fact that the myoblasts have not been subjected to ex vivo culture and that the myoblasts localizing beneath the basal lamina of single fibers were protected from the hazardous environmental stress at the time of engraftment might explain the significant improvement in function after transplantation of only 4 single fibers in the rat heart. These issues need to be studied further.

We have also demonstrated that angiogenesis is induced in infarcted hearts after single-fiber transplantation and that this is associated with increased coronary flow. This finding has been observed in previous reports with direct intramyocardial injection of skeletal myoblasts. ${ }^{12}$ In addition, other studies have shown that a nonspecific reaction caused by mechanical injury results in angiogenesis induction in the heart. ${ }^{18}$ In our study infarct size was reduced after singlefiber transplantation in the hearts that had been subjected to LCA occlusion 1 month before grafting compared with that after medium injection. One month after LCA occlusion, the myocardial scar is mature, ${ }^{11}$ and we would not expect grafting at this point to significantly reduce infarct size, although such angiogenesis might contribute to salvaging ischemic hibernating host myocardium to some extent. Apparent infarct size, as demonstrated by means of Masson trichrome staining, might have been reduced because of infiltration of the peri-infarct zone by surviving, newly generated, skeletal myoblast-derived myotubes.

It has been reported that transplantation of sliced skeletal muscle grafts into skeletal muscle itself results in extensive migration of the donor myoblasts with improved graft cell survival and fusion with host muscle. ${ }^{19}$ Although this is a simple method for obtaining a source of myoblasts for transplantation, as far as the heart is concerned, this type of graft is unlikely to be suitable because of the greater physical constraints on graft size in the heart. This will even reduce the function without perfect integration within the heart. Minced skeletal muscle grafts might be an alternative source of myoblasts for transplantation that avoids the prolonged period of in vitro culture. However, transplantation of minced muscle has recently been shown to result in failure to improve postinfarction cardiac function, possibly as a result of an exaggerated inflammatory response caused by implantation of multiple chunks of minced tissue. ${ }^{20} \mathrm{In}$ contrast, we have demonstrated that direct intramyocardial injection of isolated, living single fibers results in formation of newly generated and differentiated myotubes within the host myocardium and improves cardiac function in both doxorubicin- and myocardial infarction-induced heart failure models. These data suggest that single-fiber transplantation could be potentially useful in treating heart failure.

We thank Dr Nobushige Yamashita (First Department of Internal Medicine, Osaka University, Japan) for expert technical support in establishing the LCA ligation technique. We also thank Dr R. Kelly and Professor M. Buckingham (Department of Mo- 
lecular Biology, Institute Pasteur, France) for the gift of the 3FnlacZ-2E mouse.

\section{References}

1. Koh GY, Klug MG, Soonpa MH, Field LJ. Differentiation and longterm survival of $\mathrm{C} 2 \mathrm{C} 12$ myoblast grafts in heart. $J$ Clin Invest. 1993;92:1548-54.

2. Suzuki K, Murtuza B, Suzuki N, Smolenski RT, Yacoub MH. Intracoronary infusion of skeletal myoblasts improves cardiac function in doxorubicin-induced heart failure. Circulation. 2001;104(suppl I): 213-7.

3. Scorsin M, Hagege A, Vilquin JT, Fiszman M, Marotte F, Samuel JL, et al. Comparison of the effects of fetal cardiomyocyte and skeletal myoblast transplantation on postinfarction left ventricular function. J Thorac Cardiovasc Surg. 2000;119:1169-75.

4. Hutcheson KA, Atkins BZ, Hueman MT, Hopkins MB, Glower DD, Taylor DA. Comparison of benefits on myocardial performance of cellular cardiomyoplasty with skeletal myoblasts and fibroblasts. Cell Transplant. 2000;9:359-69.

5. Blau HM, Websrer C. Isolation and characterization of human muscle cells. Proc Natl Acad Sci U S A. 1981;78:5623-7.

6. Rosenblatt JD, Lunt AI, Pasrry J, Partridge TA. Culturing satellite cells from living single muscle fiber explants. In Vitro Cell Dev Biol. 1995;35:773-9.

7. Bischoff R. Proliferation of muscle satellite cells on intact myofibers in culture. Dev Biol. 1986;115:129-39.

8. Grounds MD, Yablonka-Reuveni Z. Molecular and cellular biology of muscle regeneration. In: Partridge T, editor. Molecular and cell biology of muscle dystrophy. London: Chapman \& Hall; 1989. p. 210-56.

9. Siveski-Iliskovic N, Hill M, Chow DA, Singal PK. Probocol protects against adriamycin cardiomyopathy without interfering with its antitumor effect. Circulation. 1995;91:10-5.
10. Iliskovic N, Singal PK. Lipid lowering: an important factor in preventing adriamycin-induced heart failure. Am J Pathol. 1997;150:72734.

11. Liu YH, Yang XP, Nass O, Sabbah HN, Peterson E, Carretero OA. Chronic heart failure induced by coronary artery ligation in Lewis inbred rats. Am J Physiol. 1997;272:H722-7.

12. Van Meter CH Jr, Claycomb WC, Delcarpio JB, Smith DM, deGruiter $\mathrm{H}$, Smart F, et al. Myoblast transplantation in the porcine model; a potential technique for myocardial repair. J Thorac Cardiovasc Surg. 1995;110:1442-8.

13. Suzuki K, Brand NJ, Smolenski RT, Jayakumar J, Murtuza B, Yacoub $\mathrm{MH}$. Development of a novel method for cell transplantation through the coronary artery. Circulation. 2000;102(suppl):III359-64.

14. Kelly R, Alonso S, Tajbakhsh S, Cossu G, Buckingham M. Myosin light chain $3 \mathrm{~F}$ regulatory sequences confer regionalized cardiac and skeletal muscle expression in transgenic mice. J Cell Biol. 1995;129: 383-96.

15. van Wijk H, Dick A, Greenough RJ, Oshodi RO, Robb D. Continuous intravenous infusion in athymic (nude) rats: an animal model for evaluation the efficiency of anti-cancer agents. Lab Anim. 2000;34: 63-9.

16. Kao RL, Chiu RCJ. Cellular cardiomyoplasty: myocardial repair with cell implantation. Austin, Tex: Medical Intelligence Unit, Landes Bioscience; 1997.

17. Qu Z, Balkir L, van Deutekom JCT, Robbins PD, Pruchnic R, Huard J. Development of approaches to improve cell survival in myoblast transfer therapy. J Cell Biol. 1998;142:1257-67.

18. Malekan R, Reynolds C, Narula N, Kelley ST, Suzuki Y, Bridges CR. Angiogenesis in transmyocardial laser revascularization. A nonspecific response to injury. Circulation. 1998;98(suppl):II62-5.

19. Fan F, Beilharz MW, Grounds MD. A potential alternative strategy for myoblast transfer therapy; the use of sliced muscle grafts. Cell Transplant. 1996;5:421-9.

20. Pouzet B, Vilquin JT, Hagege AA, Scorsin M, Messas E, Fiszman M, et al. Intramyocardial transplantation of autologous myoblasts. Can tissue processing be optimized? Circulation. 2000;102(suppl): III210-5. 